\title{
Yournal for Derson-Criented Research
}

2019; 5(2): 51-52

Published by the Scandinavian Society for Person-Oriented Research

Freely available at https://www.person-research.org

https://doi.org/10.17505/jpor.2019.05

\section{Introduction to the Special Issue:}

\section{Studying Therapeutic Change at the Level of the Individual}

This special issue is devoted to the question of how a person-oriented approach may contribute to the development of psychotherapy research. Such an approach means to study therapeutic change at the level of the individual in contrast to traditional psychotherapy research which focuses on groups rather than individuals.

One important aspect of this is a focus on measuring and analyzing variation and change over time within the individual, rather than on comparing average change between groups of patients from before to after treatment. A detailed study of individual change requires intensive longitudinal data, and an important method here is experience sampling, or ecological momentary assessment (EMA). But how willing are patients to respond to questionnaires daily, or even more often than so?

In the first article in this issue, Soyster, Bosley, Reeves, Altman and Fisher (2019) describe an investigation of the feasibility of using EMA in patients suffering from mood and anxiety disorders, PTSD, and substance abuse, when using sampling periods ranging from 7 to 30 days. They found that the participants completed more than $80 \%$ of all EMA surveys, and there was no evidence that compliance differed due to participant demographics, diagnoses, or personality characteristics.

As argued by Soyster et al. (2019), EMA offers a number of benefits over traditional assessment methods, such as greater ecological validity, the ability to study psychological phenomena as they occur in real time (instead of reporting on them retrospectively), and the collection of time-series data that make it possible to study processes over time within the individual.

In the second article, Lundh and Falkenström (2019) expand the perspective to include also other aspects of a person-oriented approach to psychotherapy research. They argue that traditional psychotherapy research is insufficiently sensitive to provide us with knowledge that can make us understand how psychotherapy works. They discuss three specific kinds of such "insensitivities": First, because traditional psychotherapy research stays at a group level of analysis it is insensitive to variation and change in the individual patient. Second, because the focus in traditional psychotherapy research is on large treatment packages, it is insensitive to the effects of specific interventions and interactions. Here they criticize traditional randomized controlled studies in psychotherapy research as representing a kind of pseudo-experimental research. Third, traditional psychotherapy research is criticized as being insensitive to the therapist and patient as individual persons, and to nuances of their interaction.

As argued by Lundh and Falkenström (2019), a fully person-oriented approach to psychotherapy research is (1) idiographic (by focusing on processes, and the relation between processes over time, within the individual); (2) nomothetic (by first striving to identify regularities and patterns of change at the level of individual treatments, and then to formulate the observed regularities into a general theory), and (3) holistic (by focusing on patient and therapist as two persons in interaction, in such a way that the integrity of the system is retained).

It may be asked how this third aspect of a personoriented approach can be realized methodologically. That is, how can we collect and analyze data on the interpersonal interaction between patient and therapist in a way that may capture essential nuances of their interaction? The third article in this special issue, which is written by Strunk and Lichtwarck-Aschoff (2019), illustrates one possible way how this might be done. They studied two therapies, where all sessions were coded by using a 10s sampling frequency that incorporated both verbal and non-verbal behaviors. The high sampling frequency resulted in long time series, which made it possible to apply non-linear analysis techniques to the therapeutic relationship between client and therapist.

Strunk and Lichtwarck-Aschoff's (2019) whole analysis is carried out within the theoretical framework of nonlinear dynamical systems. In such complex systems positive and negative feedback processes occur simultaneously; this complexity means that even if we should know all the elements of the system and how they tend to interact, longterm prediction of the system's behavior will be poor (an example of this is the so-called "butterfly effect"). Although complex non-linear systems have an infinite number of possible states, their internal dynamics will lead them to self-organize into some kind of equilibrium states, or "attractor" states. Such an attractor state can be beneficial but it can also be quite problematic (e.g., psychopathology). In this perspective successful psychotherapy is seen as changing self-organized patterns (i.e., from a 
Slofstra et al.: Redefining therapeutic outcomes of depression treatment.

problematic attractor state to a beneficial one) by a process called phase-transition (which involves critical fluctuations until the system stabilizes in the new more beneficial state). A main purpose of Strunk and Lichtwarck-Aschoff's (2019) study was to test whether the two studied therapies exhibited typical generic markers of non-linear dynamical systems, such as limited long-term predictability (i.e., butterfly effects), the occurrence of critical fluctuations as a marker for phase-transitions, and self-organized pattern formation.

The potential of dynamical systems as a model for understanding treatment processes is also present in the fourth article, written by Bosley, Soyster and Fisher (2019). In their paper they report a study of how fluctuation in positive affect (PA) and negative affect (NA) before therapy was associated with treatment response in a clinical sample of 32 patients suffering from mood and anxiety disorder. The patients completed four daily assessments of PA and NA for 30 days prior to receiving CBT. Interestingly, variability, instability, and inertia of PA were all found to be related to treatment response in the form of decreased anxiety and depression. More specifically, the results showed that higher variability in positive affect, and lower instability and inertia of positive affect, could predict positive treatment response.

Importantly, Bosley et al.'s (2019) study used EMA not to investigate therapeutic change at the level of the individual, but to measure affect dynamics at the level of the individual. Still, this is a methodology that is of potential interest also for the study of individual therapeutic change, because results such as these suggest the possibility of personalizing treatment on the basis of idiographic patterns of affect dynamics that are assessed before treatment.

In the fifth article, Slofstra, Booij, Hoenders and Castelein (2019) provide a critical reflection on how treatment outcome is typically defined in traditional research on the treatment of depression. First, they argue that the outcomes of depression treatment need to be broadened, from a focus mainly on depressive symptomatology to an increased focus on the ability to enjoy life, find meaning in life, and to feel life satisfaction. Second, because depressions tend to recur in many patients, they argue that the outcomes of depression treatment also need to be lengthened beyond the acute treatment phase by taking a life-time perspective on depression. Accordingly, they also propose adaptations to broaden and lengthen measurements in routine outcome monitoring systems to identify predictors of personalized sustainable recovery.

In the sixth and final article, Trompetter, Johnston, Johnston, Vollenbroek-Hutten and Scheurs (2019) describe an exploratory study where they used a single-case design to study processes in three patients treated for chronic pain by Acceptance and Commitment Therapy (ACT). Although they found no treatment effects of the ACT intervention in these three patients, the results showed that in two of the patients pain interference co-varied with both experiential avoidance and values-based living, in a direction that is predicted from ACT theory. Because these associations were found only on concurrent days, but not on consecutive days, however, the findings do not provide strong support for ACT theory. The paper by Trompetter et al. illustrates a kind of research design that may be used to investigate not only how different symptoms co-vary, but also how symptoms co-vary with measures of theoretically derived constructs.

Finally, many thanks for invaluable contributions to the preparation of this special issue of JPOR go to the Guest editor, Dr. Evelien Snippe, University of Groningen, the Netherlands.

\section{Lars-Gunnar Lundh}

Editor JPOR

\section{References}

Bosley, H. G., Soyster, P. D., \& Fisher. A. J. (2019). Affect dynamics as predictors of symptom severity and treatment response in mood and anxiety disorders: Evidence for specificity. Journal for Person-Oriented Research, 5(2), 101-114. https://doi.org/10.17505/jpor.2019.09

Lundh, L. G., \& Falkenström, F. (2019), Towards a personoriented approach to psychotherapy research. Journal for Person-Oriented Research, 5(2), 65-79. https://doi.org/10.17505/jpor.2019.07

Slofstra, C., Booij, S. H., Hoenders, H. J. R., \& Castelein, S. (2019), Redefining therapeutic outcomes of depression treatment. Journal for Person-Oriented Research, 5(2), 115-122. https://doi.org/10.17505/jpor.2019.10

Soyster, P. D., Bosley, H. G., Reeves, J. W., Altman, A. D., \& Fisher, A. J. (2019), Evidence for the feasibility of person-specific ecological momentary assessment across diverse populations and study designs. Journal for Person-Oriented Research, 5(2), 53-64. https://doi.org/10.17505/jpor.2019.06

Strunk, G., \& Lichtwarck-Aschoff, A. (2019). Therapeutic chaos. Journal for Person-Oriented Research, 5(2), 81-100. https://doi.org/10.17505/jpor.2019.08

Trompetter, H. R., Johnston, D. W., Johnston, M., Vollenbroek-Hutten, M. M., \& Schreurs, K. M. G. (2019). Are processes in Acceptance \& Commitment Therapy (ACT) related to chronic pain out-comes within individuals over time? An exploratory study using n-of-1 designs. Journal for Person-Oriented Research, 5(2), 123-136. https://doi.org/10.17505/jpor.2019.11 